\title{
Pharmacoinformatics elucidation of potential drug targets against migraine to target ion channel protein KCNKI8
}

This article was published in the following Dove Press journal:

Drug Design, Development and Therapy

2I May 2014

Number of times this article has been viewed

\section{Sheikh Arslan Sehgal \\ Mubashir Hassan \\ Sajid Rashid}

National Center for Bioinformatics, Quaid-i-Azam University, Islamabad, Pakistan

Video abstract

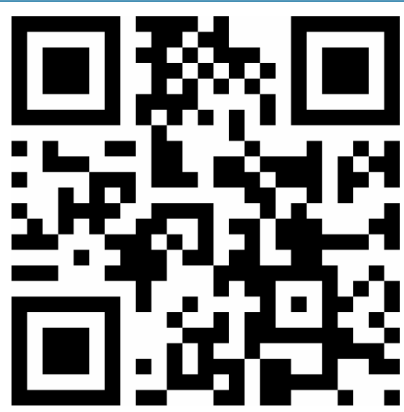

Point your SmartPhone at the code above. If you have a QR code reader the video abstract will appear. Or use: http://dvpr.es/QTrQxw
Correspondence: Sajid Rashid National Center for Bioinformatics, Quaid-i-Azam University, P.O. Box 45320, Islamabad, Pakistan Email sajidrwp@yahoo.co.uk

\begin{abstract}
Migraine, a complex debilitating neurological disorder is strongly associated with potassium channel subfamily $\mathrm{K}$ member 18 (KCNK18). Research has emphasized that high levels of KCNK1 8 may be responsible for improper functioning of neurotransmitters, resulting in neurological disorders like migraine. In the present study, a hybrid approach of molecular docking and virtual screening were followed by pharmacophore identification and structure modeling. Screening was performed using a two-dimensional similarity search against recommended migraine drugs, keeping in view the physicochemical properties of drugs. LigandScout tool was used for exploring pharmacophore properties and designing novel molecules. Here, we report the screening of four novel compounds that have showed maximum binding affinity against KCNK18, obtained through the ZINC database, and Drug and Drug-Like libraries. Docking studies revealed that Asp-46, Ile-324, Ile-44, Gly-118, Leu-338, Val-113, and Phe-41 are critical residues for receptor-ligand interaction. A virtual screening approach coupled with docking energies and druglikeness rules illustrated that ergotamine and PB-414901692 are potential inhibitor compounds for targeting KCNK18. We propose that selected compounds may be more potent than the previously listed drug analogs based on the binding energy values. Further analysis of these inhibitors through site-directed mutagenesis could be helpful for exploring the details of ligand-binding pockets. Overall, the findings of this study may be helpful for designing novel therapeutic targets to cure migraine.
\end{abstract}

Keywords: migraine, bioinformatics, modeling and docking, KCNK18, TRESK, virtual screening, pharmacoinformatics

\section{Introduction}

Migraine is a common, disabling, neurological disorder with complex neurobiology that has fascinated scientists, researchers, and physicians for decades. It was considered as vascular pathogenesis and experimental work of Wolff empowered this vascular theory. ${ }^{1}$ Wolff's results showed that an intravenous infusion of the vasoconstrictor ergotamine $[(6 \mathrm{a} R, 9 R)-N-((2 R, 5 S, 10 \mathrm{a} S, 10 \mathrm{~b} S)-5$-benzyl-10b-hydroxy-2-methyl-3,6dioxooctahydro-2H-oxazolo[3,2-a]pyrrolo[2,1-c]pyrazin-2-yl)-7-methyl-4,6,6a, 7,8,9hexahydroindolo[4,3- $f g$ ]quinoline-9-carboxamide] resulted in a decrease of temporal pulsation and headaches in migraineurs. ${ }^{2}$ Conversely, second-order trigeminal neurons in the trigeminocervical complex, which are inhibited by dihydroergotamine, suggest a neural mode of action. ${ }^{3}$ During a migraine attack, the blood vessels of the patient may be changed, but it is not to be considered as the mechanism of attack. ${ }^{4}$ Premonitory symptoms are concentration impairment, fatigue, and change in mood a day before the headache. ${ }^{5}$ 
Typical attacks are characterized by the acuity of agonizing head pain accompanied by vomiting, nausea, and aggravated by movement. The extensive dysfunction during an acute attack in sensory processing clinically manifests itself as osmophobia, phonophobia, or photophobia. Collectively, migraine is precisely explicated by dysfunction in the neuromodulatory structure of brainstems. Using positron emission tomography (PET) scans, Afridi et al have shown the activation area in the dorsolateral pons. ${ }^{4}$ Eventually, recognition of migraines as a brain disorder will have significance in investigating disorder and designing novel neural drugs.

Migraine is a debilitating brain disease. The current understanding of the underlying pathophysiology of migraine as a strange response of the central nervous system (CNS) to numerous genetic and environmental components, is inadequate. ${ }^{5,6}$ Polymorphisms and mutations in genes involved in neurotransmitter pathways and ion channels, hormonal mechanisms, and vascular functions are considered to be key factors for the susceptibility of migraine. ${ }^{7}$ Human genetics and experimental pharmacology data support a migrainebased model through activation of the trigeminovascular system and neural hyperexcitability. ${ }^{8,9}$ The prevalence of migraine is generally $3-4$ times higher among women; ${ }^{10}$ and many hypotheses have been posed for this predominance in females. ${ }^{11,12}$

Presently, the World Health Organization (WHO) recognizes migraine as a high precedence public health problem. ${ }^{13,14}$ The disabilities linked with migraine appear to be closely associated to its severity, affecting areas such as academic performance, cognitive functioning, emotional functioning, mobility, self-care, communication, ${ }^{15,16}$ sleep habits, ${ }^{17-22}$ motor coordination, ${ }^{23}$ socialization, and relationships with family members ${ }^{24,25}$ and peers. ${ }^{26}$ The main psychiatric and neurological disorders resulting from migraine are epilepsy, depression, somnolence, and anxiety disorders. ${ }^{27}$ In clinical pediatric practices, numerous alternative therapies and treatments have been suggested, such as improving sleep hygiene, ${ }^{28,29}$ using nutraceuticals; ${ }^{30,31}$ weight loss; generic psychological interventions; psychotherapy; and alleviating comorbidities in patients. ${ }^{32-34}$ Esposito et al suggested self-esteem level as an objective tool for comparing controls with children affected with migraine without aura. ${ }^{35}$ A rehabilitative device, the Nintendo Wii Fit Plus ${ }^{\mathrm{TM}}$ system (Kyoto, Japan) for improving balance and visuomotor skills impairment has had positive effects among children affected by migraine without aura. ${ }^{36}$ A personality ${ }^{37}$ and temperamental characteristics ${ }^{38}$ assessment of mothers has resulted in better clinical and comprehensive management for migraineurs' children.

Moreover, from the last 2 decades, much attention has been given to the relevance of psychiatric comorbidities, and neurological and psychological problems..$^{39-46}$ The pathophysiology of migraine implicates vascular and neurological mechanisms. Recent studies suggest that the trigeminovascular system exhibits an important role in migraine disorder ${ }^{47-49}$ due to its decisive interaction with meningeal vasculature by various transporters, peptides, and neurotransmitters located in the trigeminovascular system. Glutamate, dopamine, and serotonin are the neurotransmitters implicated in the pathogenesis of migraine. Any imbalance in these neurological systems may lead to higher migraine susceptibility. Presently, dopamine and serotonin remain under discussion regarding neurotransmitter circuits in associated case-control studies examining polymorphisms in receptors and transporters of neurological systems..$^{50,51}$

Migraine disorder has strong inherited components, which suggests the involvement of glutamate pathways in its pathogenesis. ${ }^{52}$ Glutamate is a commonly implicated element in migraine pathophysiology and may act via cortical spreading depression, central sensitization, or trigeminovascular activation. Various pharmacological and biochemical studies have also associated glutamate with migraine. Glutamate is unable to cross the blood-brain barrier and is a nonessential amino acid; it must be synthesized inside neurons from local precursors. ${ }^{53}$ Glutamate is found in related structures of neurons including the thalamus, the trigeminocervical complex, and the trigeminal ganglion. ${ }^{54}$ It affects numerous brain-specific metabolic outcomes that involve glutathione, such as the formation of glutamine $\gamma$-aminobutyric acid (GABA), incorporation into proteins, and oxidation via the tricarboxylic acid cycle (Krebs cycle) for energy. ${ }^{55}$ Generally, large amounts of glutamate are present in the brain and stored intracellularly. ${ }^{55}$ It is continuously cycled between glial cells and neurons under normal conditions known as the glutamateglutamine cycle. ${ }^{55}$ Migraine genetics understanding is important due to its relative novelty in the disease system. The recent identification of potassium channel subfamily $\mathrm{K}$ member 18 (KCNK18) functional mutation was a great success. KCNK18 is a key player gene that is associated with migraine; it consists of three exons with a $12.8 \mathrm{~kb}$ span and is mapped on chromosome 10q25.3. ${ }^{56}$ It encodes TWIK-related spinal cord potassium channel (TRESK), a potassium channel subfamily K member 18 expressed throughout the CNS and also in the trigeminal ganglion neurons. ${ }^{57}$ Numerous mutations in KCNK18 were reported using the candidate gene 
approach and large cohort case-control functional analysis. The significantly identified variant of migraine was by frameshift mutation (F129WfsX24), which perfectly segregated in the affected family of migraine with aura. This mutation protein regulates neuronal excibility and is involved in pain pathways. It is the identified genetic mutation associated with common migraine. ${ }^{58}$

Ion channels are macromolecule protein complexes and potassium channels are classified by ion type channels. Ion channel genes play a vital role in performing normal CNS functioning. These ion channels control muscle contraction, hormones, release of neurotransmitters, and significant biological functions. ${ }^{59}$ These are located in the lipid bilayer membrane and help during the movement of ions across the hydrophobic barrier that separates the cytoplasm from intracellular and extracellular organelles. ${ }^{60}$ The function of KCNK18 is to cure the outward potassium channels and produces activated outward current rectifier $\mathrm{K}^{+}$. It may function as a potassium channel that maintains the resting potential membrane. Calcium channels directly stimulate the activity of channels activated by the G(q)-protein-coupled receptor pathway. The calcium signal activates the channel via calcineurin and 14-3-3/YWHAH anchoring, which interferes with the current returned from the resting state after activation. It is inhibited by unsaturated free naturally occurring fatty acids and arachidonic acid. The activity of the channel is enhanced by isoflurane and volatile anesthetics. ${ }^{61,62}$

The current study demonstrates pharmacophore-based virtual screening to reveal novel inhibitors against migraine. Pharmacophore-based molecule libraries were screened by a two-dimensional similarity search against recommended migraine drugs. The novel molecules of diverse structural entities and common structural features were investigated. As experimental validation of KCNK18 using X-ray crystallography and nuclear magnetic resonance (NMR) is not yet available, the three-dimensional structure of KCNK18 was predicted using the crystal structure of the human two-pore domain potassium ion channel K2P1 (TWIK-1 [PDB ID: $3 \mathrm{UKM}])$. The inclusive in silico analysis may provide evidence for a reliable framework that could assist medicinal chemists for the design and development of novel molecules for potential drugs that target migraine.

\section{Materials and methods}

In the present work, sequence comparison, structure prediction, library screening, pharmacoinformatics analysis, and docking studies were performed on an HP Core-i-5 workstation (Hewlett-Packard Company, Palo Alto, CA,
USA). The amino acid sequence of KCNK18 (384 residues) was retrieved for homology modeling as the suspected gene is a candidate of migraine disorder with and without aura. ${ }^{63}$ The amino acid sequence was retrieved in FASTA format from the Uniprot Knowledge base with the accession number Q7Z418. The retrieved amino acid sequence of KCNK18 was subjected to a protein-protein basic local alignment search tool (BLAST) search against the Protein Data Bank (PDB) ${ }^{64}$ for the identification of a suitable template structure. The crystal structure of the human two-pore domain potassium ion channel K2P1 (TWIK-1) was selected as a suitable template with $40 \%$ identity, $31 \%$ query coverage, and an E-value of $4 \mathrm{e}^{-09}$. The automated protein modeling program MODELLER 9v10 $0^{65}$ was used to predict the 3D structure of KCNK18 by satisfying spatial restraints. The evaluation tools ERRAT, ${ }^{66}$ Anolea, ${ }^{67}$ ProCheck, ${ }^{68}$ and Rampage ${ }^{69}$ were applied to assess the predicted three-dimensional model of KCNK18. The structure was further evaluated by MolProbity. ${ }^{70}$ Finally, poor rotamers and Ramachandran outliers were corrected by employing WinCoot ${ }^{71}$ tool.

Numerous tools and servers were utilized to design novel compounds that might potentially inhibit KCNK18 by interacting with its predicted structure such as AutoDock, Chimera, ${ }^{72}$ VMD, PyMOL, Cresset, VegaZZ,${ }^{73}$ Chemdraw, ${ }^{74}$ mCule, Molinspiration, and Osiris Property Explorer. Docking studies were done by AutoDock tool. ${ }^{75}$ The number of rotatable bonds, $\mathrm{H}$-bond acceptors, and H-bond donors were obtained using Cresset, mCule, Molinspiration, ${ }^{76}$ and PubChem. ${ }^{77}$ The online tool Osiris Property Explorer was employed to estimate their possible tumorigenic, reproductive, or mutagenic risks and to calculate the drug-like properties of known drugs and novel designed molecules. Lipinski's rule of five was analyzed using the Cresset and mCule servers. The druglikeness values calculated by the Osiris software are positive when fragments of designed molecules are frequently present in approved drugs. The mCule, Cresset, and Osiris programs were employed to estimate the mutagenesis of novel molecules and no mutagenic risks were detected.

The properties of already known drugs for migraine were used for library screening and designing novel molecules. No ligands were found for KCNK18 in the literature and known biological databases. The aim of docking analysis was to identify the binding pattern and the relative binding specificities.

Pharmacophoric screening of compounds was performed using the LigandScout ${ }^{78}$ tool. The known biomolecules and 
three compound libraries (ZINC, Drug, and DrugLike) were screened against the structure of KCNK18. The screening procedure was performed using the default parameters. The high pharmacophore score compounds were extracted and docking analysis was carried out on the top hits. Interactions were elucidated using AutoDock. The grid box was used to define the screening site. Four pharmacophore-based compounds with optimum binding energies were selected and visualized by Chimera (v1.6) and Ligplot. ${ }^{79}$

The geometrical optimization and energy minimization of the three-dimensional structures of the designed molecules were performed by Vega ZZ, LigandScout, and ChemDraw Ultra. The results were analyzed using AutoDock tools, Chimera 1.6, and Ligplot.

\section{Results and discussion}

The aim of this research was based on the relationship between KCNK18 and migraine and its bioinformatics analysis for designing, identifying, and evaluating novel inhibitors. The top five optimally aligned templates with total scores, E-values, maximum identity, and query coverage are mentioned in Table 1. The 3UKM template gave the best evaluation results. The three-dimensional structure of KCNK18 (Figure 1) was comparatively modeled using the crystal structure of 3UKM as a modeling template.

The evaluation tools showed the efficacy and reliability of the predicted structure of KCNK $18,90.05 \%$ of the favored region was detected in the Ramachandran plot. Only eleven residues out of 384 were observed in the outlier region. Subsequently, outliers and all the poor rotamers were corrected to refine the predicted model of KCNK18.

Experimental analysis revealed that the drugs (Figure 2) used in the current analysis have significant values (Table 2) to cure migraine; their two-dimensional structures are shown in Figure 2. However, the docking analysis of the selected

Table I Five BLAST aligned templates of KCNK I8 with E-value, query coverage, and identity

\begin{tabular}{lllll}
\hline PDB ID & $\begin{array}{l}\text { Total } \\
\text { score }\end{array}$ & $\begin{array}{l}\text { Query } \\
\text { coverage }\end{array}$ & E-value & $\begin{array}{l}\text { Maximum } \\
\text { identity }\end{array}$ \\
\hline 3UKM & 125 & $40 \%$ & $4 \mathrm{e}-09$ & $31 \%$ \\
4BW5 & 108 & $54 \%$ & $2 \mathrm{e}-15$ & $35 \%$ \\
3UM7 & 118 & $29 \%$ & $1 \mathrm{e}-07$ & $41 \%$ \\
3T4D & 68.2 & $21 \%$ & 0.002 & $43 \%$ \\
3TIC & 67.0 & $21 \%$ & 0.005 & $40 \%$ \\
\hline
\end{tabular}

Abbreviations: BLAST, basic local alignment search tool; KCNKI8, potassium channel subfamily K member I8; PDB ID, Protein Data Bank identification.

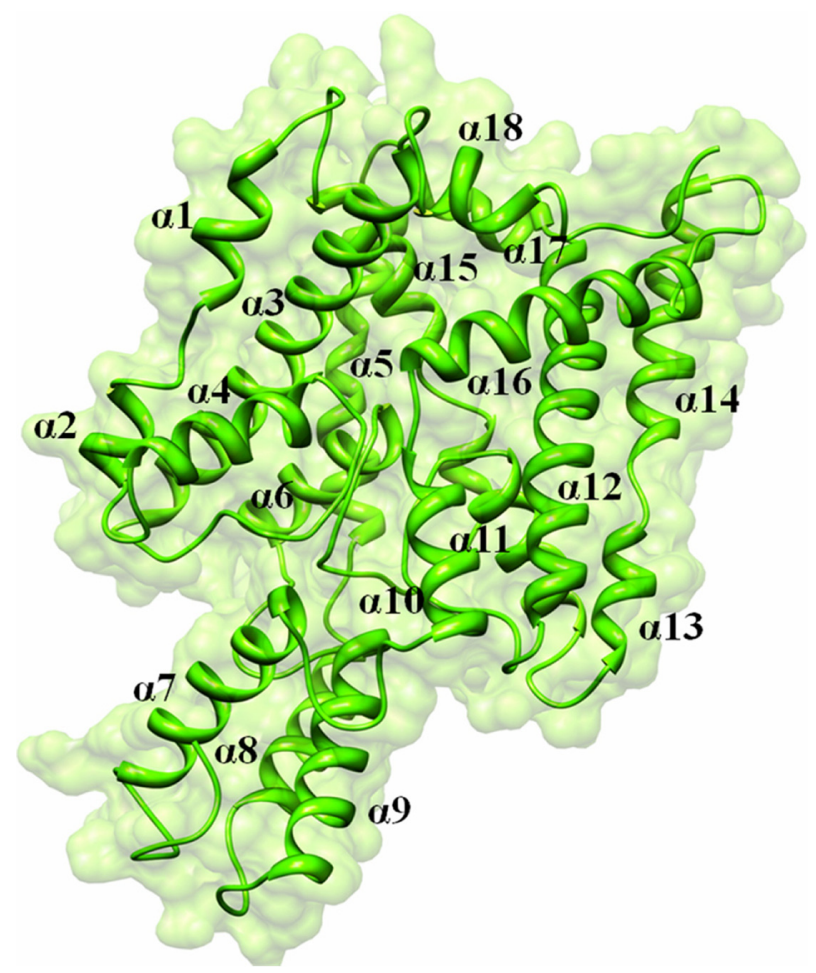

Figure I KCNKI8 structure predicted by utilizing PDB ID 3UKM.

Abbreviations: KCNKI8, potassium channel subfamily K member I8; PDB ID, Protein Data Bank identification.

drugs revealed variations in their binding energies. Initially, docking analysis was performed with 150 runs and ten poses were saved, out of which the best poses with the lowest binding energy was chosen for each compound. Our results indicate that nine selected compounds (almotriptan, aspirin, ibuprofen, acetaminophen, diclofenac, rizatriptan, lasmiditan, ergotamine, telcagepant) efficiently bind to KCNK18 (Table 2).

All the nine selected drugs were analyzed on the basis of binding energy values and the drug properties mentioned in Table 2. The selected drugs have cyclic molecules with significant biological properties. These molecules are potential anti-migrainic agents. The lowest binding energy was observed in ergotamine among all the selected drugs that showed good ligand properties.

Next, libraries were screened by LigandScout. After screening each library, 17 compounds with the lowest pharmacophore scores $(>50)$ were chosen. AutoDock tools were utilized for docking analysis and the top four molecules with the lowest binding energies were selected for further analysis (Figure 3). This study revealed reliable results by docked analysis of selected compounds with KCNK18. The four scrutinized molecules from a combination of all the selected 

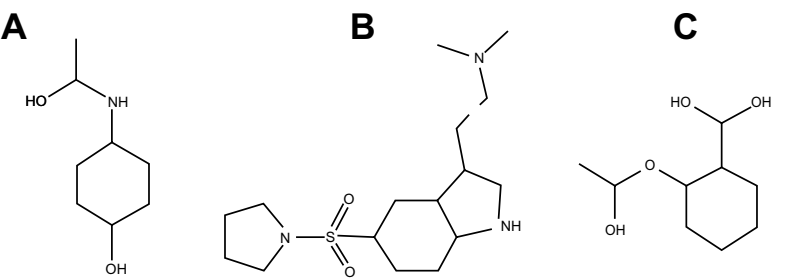

$\mathbf{E}$

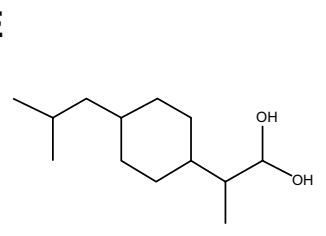

$\mathbf{F}$
$\mathbf{G}$

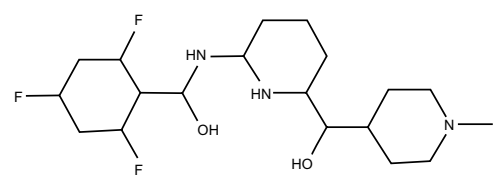

I
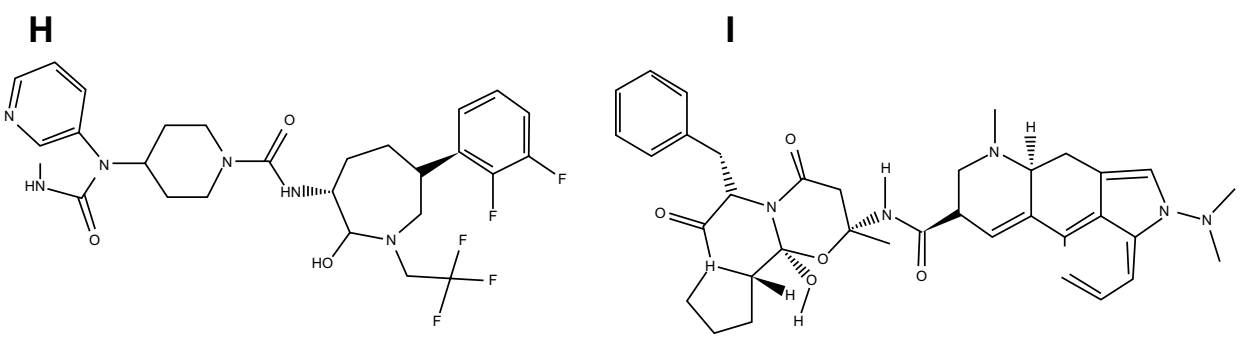

Figure 2 Two-dimensional structures of investigated drugs.

Notes: (A) acetaminophen; (B) almotriptan; (C) aspirin; (D) diclofenac; (E) ibuprofen; (F) rizatriptan; (G) lasmiditan; (H) telcagepant; (I) ergotamine.

libraries were elucidated (Table 3). It was also observed and analyzed that the majority of the compounds would bind at the binding residues between Asp-46 and Leu-338. The docking analysis revealed that novel and known molecules bind at around the same residues and revealed the binding pocket (Figure 4). Another observation is that selected molecules occupied the space between Thr-32 to Gly-118 and Cys-253 to Glu-332. It is also possible that the combination of observed binding residues may lead to the lower binding energy achieved in the current study.

The highest ranked ligand from all libraries was elucidated, namely PB-414901692 from pharmacological-based screening. It was observed that Asp-46, Ile-44, Ile-324, Gly118, Phe-41, Thr-33, Thr-32, Cys-253, Glu-332, Phe-326, Asp-328, Gly-327, Tle-324, and Met-225 exhibited good binding interactions with all the docked ligands. In an effort to better comprehend the interactions between ligand and amino acid residues in the active site of the protein, a plot of amino acids-ligand interactions was generated using Ligplot and Chimera (v1.6) as shown in Figure 5.

Non-steroidal anti-inflammatory drug treatment was not very successful. Ergotamine is a famous pharmacological remedy applied in obstetrics and neurology. Migraineurs improperly taking ergotamine may increase the risk of coronary events, ergotism, and other health problems due to the potential interactions. ${ }^{80}$ The long-term use of ergotamine for the treatment of migraine is associated with cardiac fibrotic and pleuropulmonary reactions. It causes blood vessel constriction through blockage of the alpha-receptors and stimulation of the serotonin-receptors on blood vessel walls in the peripheral circulation and CNS. Usually, the migraine patients have been found to use ergotamine as a drug for the relief of migraine attack and pain. ${ }^{81}$ Treatment of migraine with colchicines was tentatively successful with inflammatory parameters, cough, fever, and resulted in complete resolution of pleural fluids. ${ }^{82}$

Lasmiditan (2,4,6-trifluoro- $N$-[6-[(1-methylpiperidin4-yl)carbonyl]pyridin-2-yl]benzamide) is a highly selective serotonin (5-HT) 5-HT(1F) and high-affinity receptor agonist. It has a unique scaffold of pyridinoyl-piperidine that is not present in any other class of anti-migraine drugs. Its pharmacological profile and chemical structure differentiates it from the triptans. ${ }^{83}$

Oral diclofenac is an effective medicine for the treatment of acute migraine, providing relief from migraine pain and associated symptoms. Mostly, the adverse events are transient and mild. ${ }^{79}$ Aspirin is another effective acute migraine pain treatment, similar to sumatriptan. ${ }^{84}$

In general anesthesia and pain pathways, a role has been proposed for TRESK encoded by KCNK $18 .{ }^{58}$ Lafrenière 


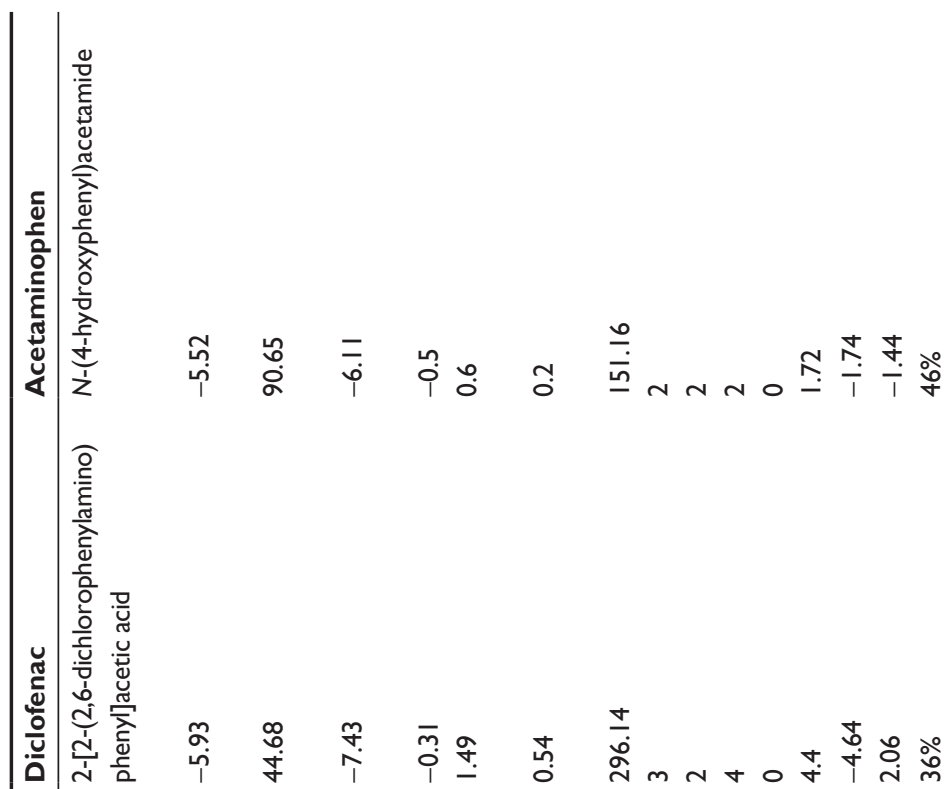

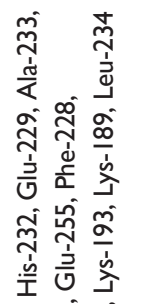

ำ ㅇำ

要要

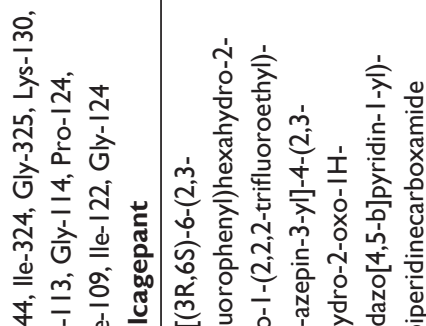

高旁喜
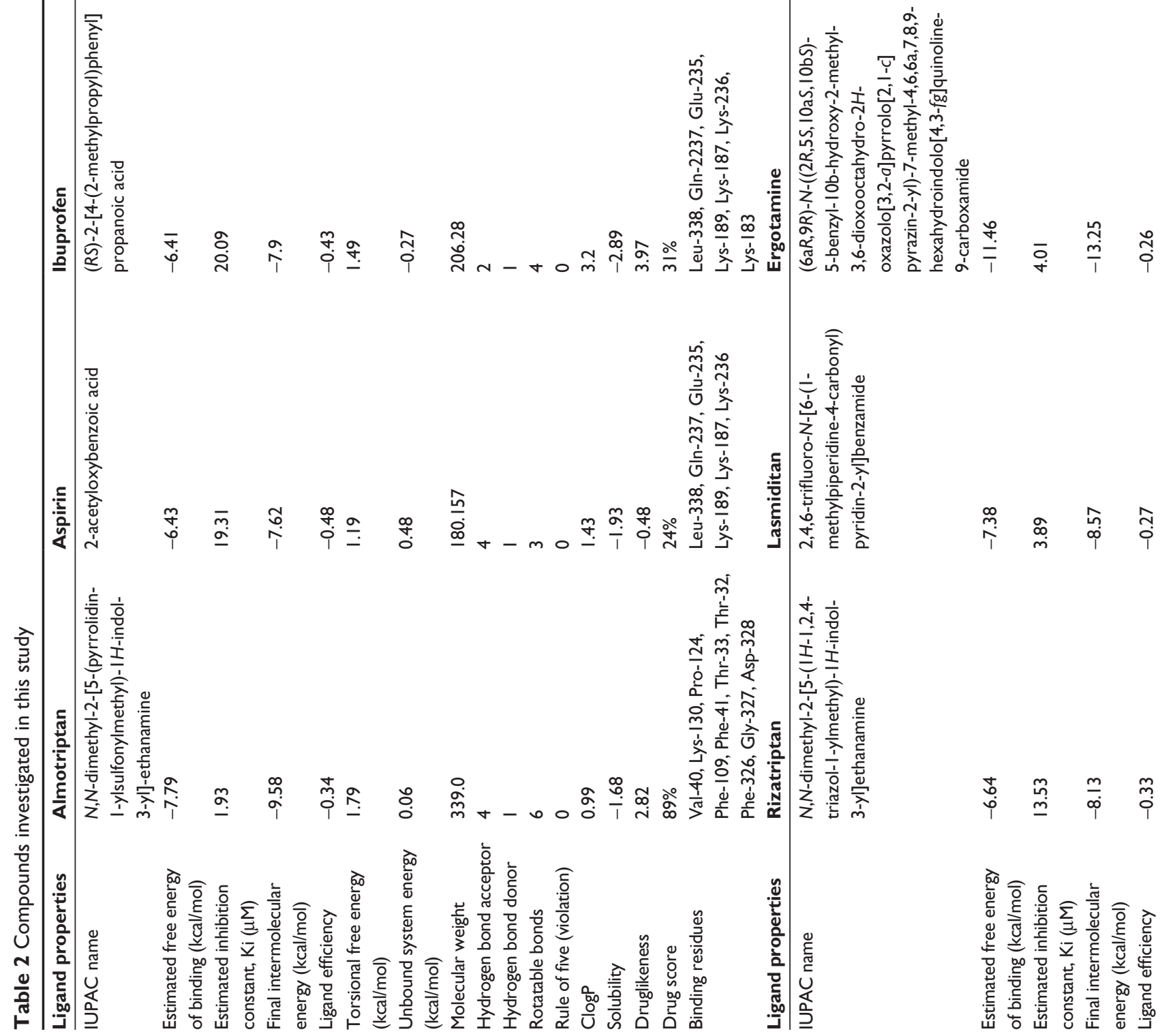


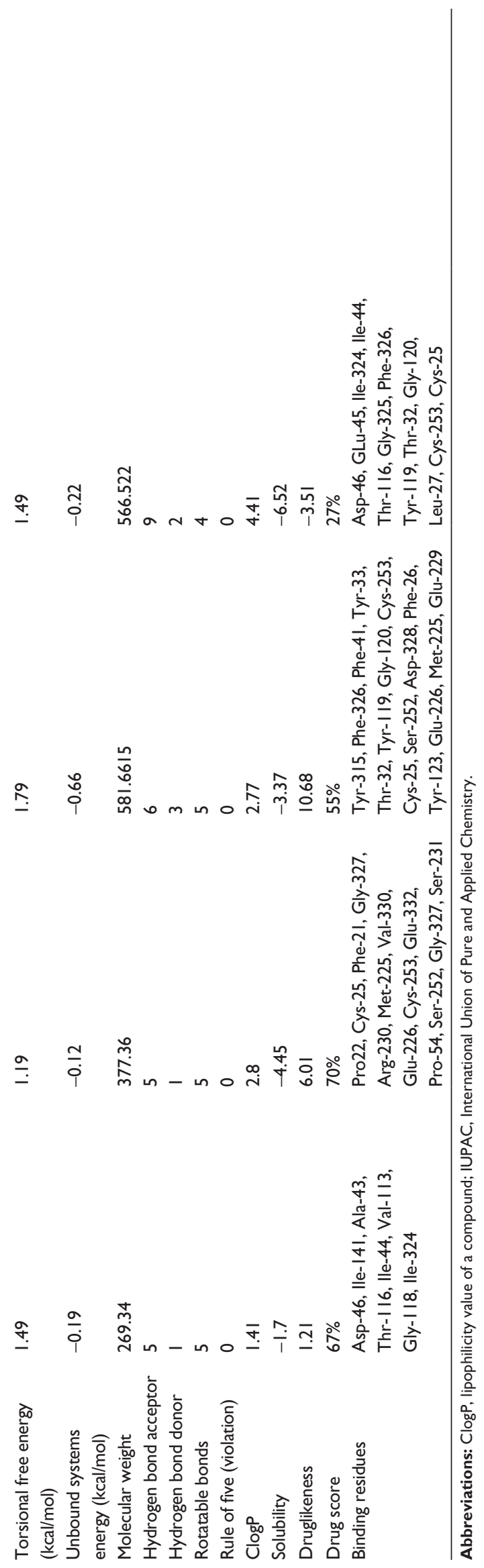

et al suggested the involvement of TRESK by screening the $K C N K 18$ gene in subjects diagnosed with migraine. ${ }^{59}$ A frameshift mutation reported (F139WFSx24) that perfectly segregates in large pedigree with typical migraine with aura and prominently identified TRESK expression in the sensory ganglia, trigeminal ganglion, and migraine salient areas.

Functional depiction of this frameshift mutation revealed that TRESK loses its function completely and the mutant subunit suppresses the function channel of wild-type protein through a negative dominant effect, consequently explicating the dominant penetrance of this allele. These observations thus support the role of TRESK in typical migraine with aura pathogenesis and support further the role of this channel as a potential therapeutic target. ${ }^{58}$

In this study, in silico methodologies such as homology modeling, comparative pharmacoinformatics, docking analyses, and structural and pharmacophore library screening were carried out. The three-dimensional structure of KCNK18 was modeled by employing a crystal structure template. The predicted structure has a good degree of accuracy, especially at the active site of the protein. Comparative docking was tested by automated docking analysis using AutoDock Tools, which has allowed us to reveal the ligand-receptor interactions of the most representative drugs for migraine. A detailed comparative docking analysis of the interactions between KCNK18 and drug molecules has pointed out the best compound with the lowest binding energy that can be taken into account for the novel drug designing. The results of the docking analysis suggest that an ideal ligand must be one that satisfies its druglikeness parameters and has the lowest docking score. Coinciding with this statement, with the satisfaction of the parameters of lowest docking energy, toxicity, drug score, and Lipinski's rule of five, it is suggested that ergotamine targets KCNK18 and is a potential drug molecule for migraine treatment. The ZINC, Drug, and DrugLike libraries were screened for pharmacophoric similarity to KCNK18. The scrutinized compounds using various libraries were analyzed for their binding properties. Our docking results revealed the involvement of Asp-46, Ile-324, Ile-44, Gly-118, Leu-338, Val-113, and Phe-41 residues and mutational studies of these residues could be highly effective in further studies. The top four scrutinized compounds from the current work had the lowest binding energies. It stands to reason that the discovered compounds in this work have the propensity to be good candidates for the treatment of migraine by targeting KCNK18. The proposed and used strategies have led to a simplification of the process 
<smiles>OS(O)(NCC1CCCCC1COCC1CCCCC1)C1CCC(F)CC1Cl</smiles>

C<smiles>COC1CCC(CN2C(O)C3CCCCC3C(CC3CSNN3)C2O)CC1</smiles><smiles>[B]C1CCCCC1N1NC(C(O)NC2CCCCC2N2CC(Br)CN2)C(O)CC1C</smiles><smiles>CC1CCCC(N2C(O)C3CCCCC3C(CC3CSNN3)C2O)C1</smiles>

Figure 3 Novel molecules in two-dimensional structures.

Notes: (A) PB-4083 I8540; (B) PB-4I50|90। 0; (C) PB-4|490|730; (D) PB-4I 4901692.

of novel drug designing as biological investigation and drug analysis can be carried out on candidates with good results without the need to waste money and time on poor activity compounds.

In conclusion, this analysis suggests that the selected drugs and novel compounds are efficacious in the treatment of acute migraine attacks. Though numerous differences exist between baseline population and trail methodology studies, and computational pharmacoanalysis, we may be justified in concluding that ergotamine may be a good option for the treatment of migraine. Considering these findings, further studies and synthesis of these novel compounds may result in similar response rates and help to cure migraine pains.

\section{Acknowledgments}

We are grateful to Miss Shagufta Shafique and Miss Saima Younas (Functional Informatics Laboratory, Quaid-i-Azam University, Islamabad, Pakistan) for their kind assistance.

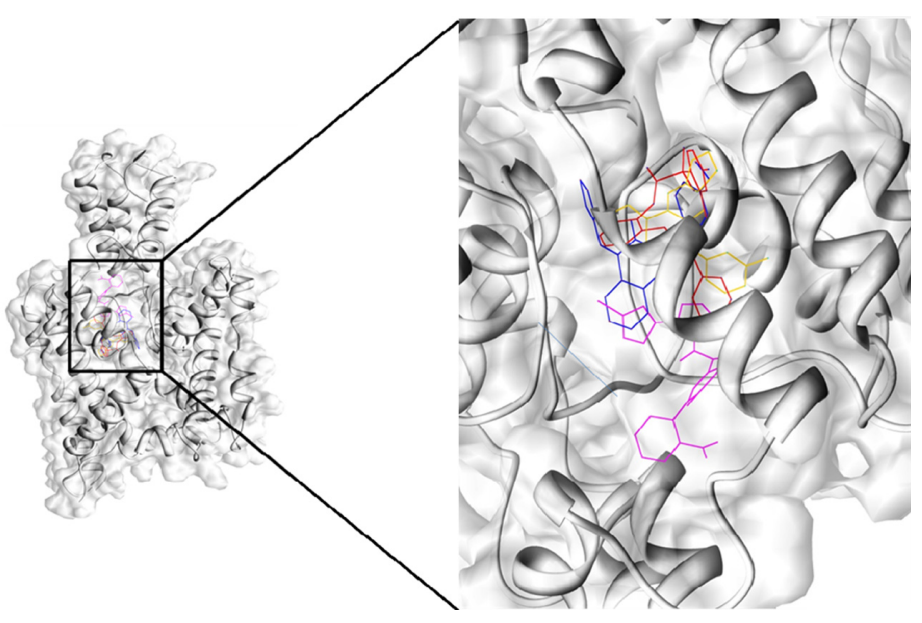

Figure 4 All the selected ligands bind at same binding pocket in KCNKI8. Abbreviation: KCNKI8, potassium channel subfamily K member 18. 
Table 3 Ligand properties and bioinformative detail of the top four screened compounds in the present study

\begin{tabular}{|c|c|c|c|c|}
\hline Ligand properties & PB-4083 I 8540 & PB-4I5019010 & PB-4I490I730 & PB-4I490I692 \\
\hline Estimated free energy of binding $(\mathrm{kcal} / \mathrm{mol})$ & -9.07 & -9.19 & -9.66 & -9.79 \\
\hline Estimated inhibition constant, $\mathrm{Ki}(\mu \mathrm{M})$ & 226.18 & 183.0 & 82.92 & 66.95 \\
\hline Final intermolecular energy ( $\mathrm{kcal} / \mathrm{mol})$ & -11.45 & 10.68 & -10.85 & -10.38 \\
\hline Ligand efficiency & -0.32 & -0.29 & -0.36 & -0.39 \\
\hline Unbound system energy (kcal/mol) & -0.07 & -0.36 & -0.75 & -0.2 \\
\hline Molecular weight & 440 & 534 & 399 & 369 \\
\hline LogP & 6.5 & 1.82 & 3.67 & 3.0 \\
\hline Hydrogen bond acceptor & 4 & 10 & 6 & 5 \\
\hline Hydrogen bond donor & 3 & 7 & 4 & 4 \\
\hline Rotatable bonds & 8 & 6 & 5 & 3 \\
\hline Rule of five (violation) & 0 & 0 & 0 & 0 \\
\hline $\mathrm{Clog} P$ & -0.98 & -4.11 & 1.18 & 1.68 \\
\hline Solubility & $-5.6 \mathrm{I}$ & -2.99 & -3.17 & -3.55 \\
\hline Druglikeness & -5.7 & -5.7 & -0.83 & -2.4 \\
\hline Drug score & $28 \%$ & $33 \%$ & $53 \%$ & $44 \%$ \\
\hline Binding residues & $\begin{array}{l}\text { Asp-46, Ala-43, } \\
\text { Ile-324, Leu-I38, } \\
\text { Ile-I4I, Ile-44, } \\
\text { Met-I34, Thr-I I6, } \\
\text { Ala-I37, Val-II7, } \\
\text { Gly-II8 }\end{array}$ & $\begin{array}{l}\text { Val-40, Ile-44, Lys-I30, } \\
\text { Val-I I3, Phe-109, } \\
\text { Ile-122, Gly-I20, } \\
\text { Gly-I I8, Gly-325, } \\
\text { Gly-37, Phe-4I, Tyr-33, } \\
\text { Thr-32, Phe-326 }\end{array}$ & $\begin{array}{l}\text { Asp-46, Tle-324, } \\
\text { Ala-43, Ile-44, } \\
\text { Met-I34, Gly-I I8, } \\
\text { Ala-I37, Val-I7, } \\
\text { Thr-II6, Leu-I38, } \\
\text { Ile-I4I }\end{array}$ & $\begin{array}{l}\text { Phe-109, Val-I I3, } \\
\text { Gly-I I8, Lys-130, } \\
\text { Val-40, Tke-44, } \\
\text { Tle-324, Gly-325, } \\
\text { Phe-326, Gly-37, } \\
\text { Phe-4I, Thr-32, Tyr-33 }\end{array}$ \\
\hline
\end{tabular}

Abbreviations: ClogP, lipophilicity value of a compound; LogP, lipophilicity measurement.
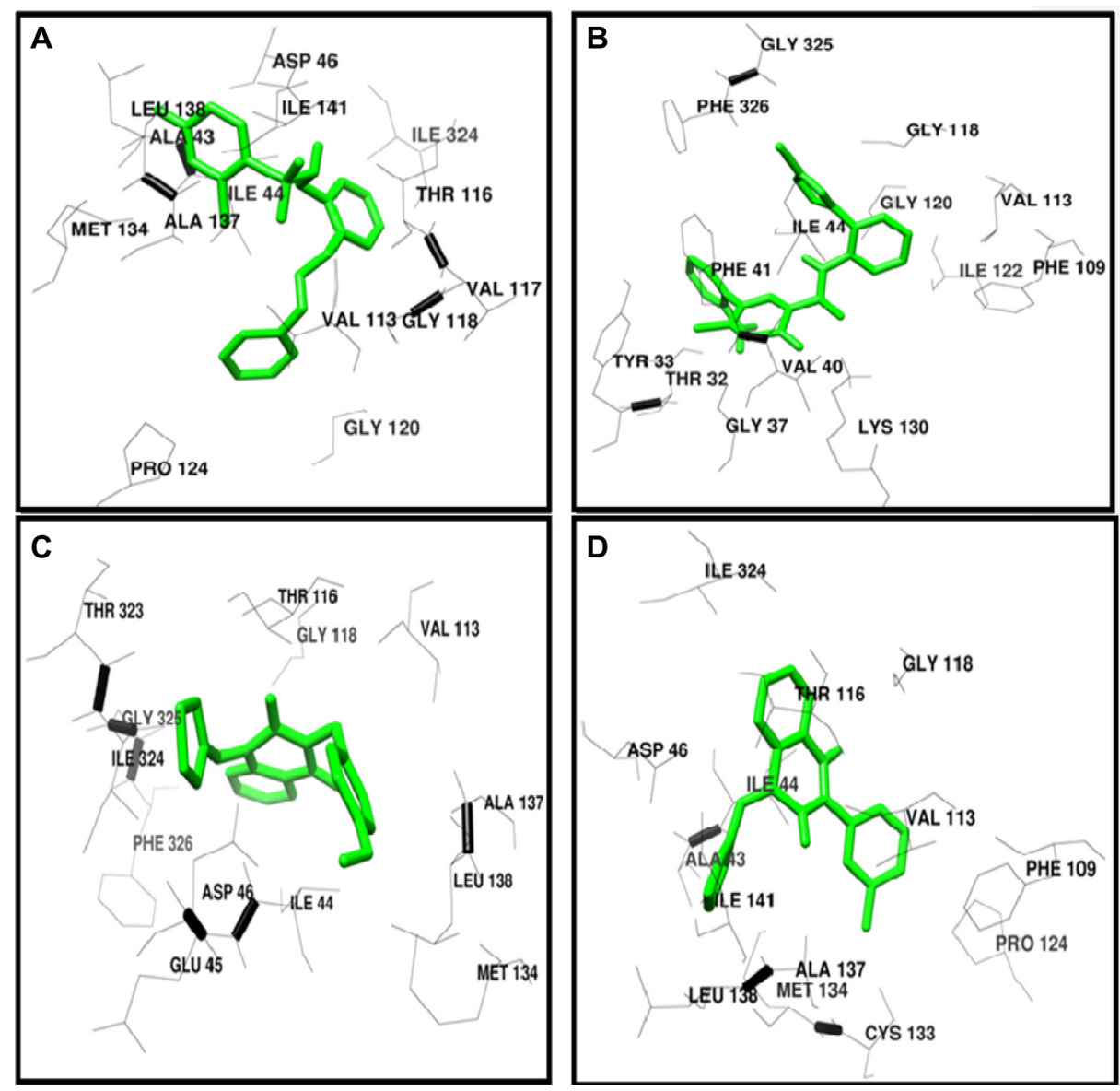

Figure 5 Binding pocket and interacting residues of novel analyzed molecules.

Notes: Binding site pattern of (A) PB-4083 I8540; (B) PB-4150190 I0; (C) PB-41490 I730; (D) PB-414901692 with KCNK 18. Ligand is shown in green color. Binding residues of KCNKI 8 are shown in black wires. 


\section{Disclosure}

The authors report no conflicts of interest in this work.

\section{References}

1. Wolff H. Headache and Other Head Pain. New York: Oxford University Press; 1948.

2. Hoskin KL, Kraube H, Goadsby PJ. Central activation of the trigeminovascular pathway in the cat is inhibited by dihydroergotamine. A c-Fos and electrophysiological study. Brain. 1996;119(1):249-256.

3. Limmroth V, May A, Auerbach P, Wosnitza G, Eppe T, Diener HC. Changes in cerebral blood flow velocity after treatment with sumatriptan or placebo and implications for the pathophysiology of migraine. J Neurol Sci. 1996;138(1-2):60-65.

4. Afridi SK, Matharu MS, Lee L, et al. A PET study exploring the laterality of brainstem activation in migraine using glyceryl trinitrate. Brain. 2005;128(Pt 4):932-939.

5. Kelman L. The triggers or precipitants of the acute migraine attack. Cephalalgia. 2007;27(5):394-402.

6. Wessman M, Terwindt GM, Kaunisto MA, Palotie A, Ophoff RA. Migraine: a complex genetic disorder. Lancet Neurol. 2007;6(6): 521-532.

7. Montagna P. Migraine: a genetic disease? Neurol Sci 29 Suppl 1. 2008; S47-S51.

8. Striessnig J. Pathophysiology of migraine headache: insight from pharmacology and genetics. Drug Discov Today. 2005;2(4):453-462.

9. de Vries B, Frants RR, Ferrari MD, van den Maagdenberg AM. Molecular genetics of migraine. Hum Genet. 2009;126(1):115-132.

10. Lipton RB, Bigal ME. The epidemiology of migraine. Am J Med. 2005; 118 Suppl 1:3S-10S.

11. Aloisi AM. Gonadal hormones and sex differences in pain reactivity. Clin J Pain. 2003;19(3):168-174.

12. Low NC, Cui L, Merikangas KR. Sex differences in the transmission of migraine. Cephalalgia. 2007;27(8):935-942.

13. Leonardi M, Steiner TJ, Scher AT, Lipton RB. The global burden of migraine: measuring disability in headache disorders with WHO's Classification of Functioning, Disability and Health (ICF). J Headache Pain. 2005;6(6):429-440.

14. Vos T, Flaxman AD, Naghavi M, et al. Years lived with disability (YLDs) for 1160 sequelae of 289 diseases and injuries 1990-2010: a systematic analysis for the Global Burden of Disease Study 2010. Lancet. 2012;380(9859):2163-2196.

15. Kernick D, Campbell J. Measuring the impact of headache in children: a critical review of the literature. Cephalalgia. 2009;29(1):3-16.

16. Esposito M, Pascotto A, Gallai B, et al. Can headache impair intellectual abilities in children? An observational study. Neuropsychiatr Dis Treat. 2012;8:509-513.

17. Carotenuto M, Guidetti V, Ruju F, Galli F, Tagliente FR, Pascotto A. Headache disorders as risk factors for sleep disturbances in school aged children. J Headache Pain. 2005;6(4):268-270.

18. Vendrame M, Kaleyias J, Valencia I, Legido A, Kothare SV. Polysomnographic findings in children with headaches. Pediatr Neurol. 2008;39(1):6-11.

19. Carotenuto M, Esposito M, Precenzano F, Castaldo L, Roccella M. Cosleeping in childhood migraine. Minerva Pediatr. 2011;63(2): 105-109.

20. Carotenuto M, Esposito M, Pascotto A. Migraine and enuresis in children: an unusual correlation? Med Hypotheses. 2010;75(1):120-122.

21. Esposito M, Gallai B, Parisi L, et al. Primary nocturnal enuresis as a risk factor for sleep disorders: an observational questionnaire-based multicenter study. Neuropsychiatr Dis Treat. 2013;9:437-443.

22. Esposito M, Roccella M, Parisi L, Gallai B, Carotenuto M. Hypersomnia in children affected by migraine without aura: a questionnaire-based case-control study. Neuropsychiatr Dis Treat. 2013;9:289-294.

23. Esposito M, Verrotti A, Gimigliano F, et al. Motor coordination impairment and migraine in children: a new comorbidity? Eur J Pediatr. 2012;171(11):1599-1604.
24. Lipton RB, Bigal ME, Kolodner K, Stewart WF, Liberman JN, Steiner TJ. The family impact of migraine: population-based studies in the USA and UK. Cephalalgia. 2003;23(6):429-440.

25. Esposito M, Gallai B, Parisi L, et al. Maternal stress and childhood migraine: a new perspective on management. Neuropsychiatr Dis Treat. 2013;9:351-355.

26. Verrotti A, Agostinelli S, D'Egidio C, et al. Impact of a weight loss program on migraine in obese adolescents. Eur J Neurol. 2013;20(2): 394-397.

27. Bellini B, Arruda M, Cescut A, et al. Headache and comorbidity in children and adolescents. J Headache Pain. 2013;14(1):1-11.

28. Bruni O, Galli F, Guidetti V. Sleep hygiene and migraine in children and adolescents. Cephalalgia. 1999;19 Suppl 25:57-59.

29. Carotenuto M, Gallai B, Parisi L, Roccella M, Esposito M. Acupressure therapy for insomnia in adolescents: a polysomnographic study. Neuropsychiatr Dis Treat. 2013;9:157-162.

30. Esposito M, Ruberto M, Pascotto A, Carotenuto M. Nutraceutical preparations in childhood migraine prophylaxis: effects on headache outcomes including disability and behaviour. Neurol Sci. 2012;33(6): 1365-1368.

31. Esposito M, Carotenuto M. Ginkgolide B complex efficacy for brief prophylaxis of migraine in school-aged children: an open-label study. Neurol Sci. 2011;32(1):79-81.

32. Chopra R, Robert T, Watson DB. Non-pharmacological and pharmacological prevention of episodic migraine and chronic daily headache. WV Med J. 2012;108(3):88-91.

33. Kröner-Herwig B, Gassmann J. Headache disorders in children and adolescents: their association with psychological, behavioral, and socio-environmental factors. Headache. 2012;52(9):1387-1401.

34. Sieberg CB, Huguet A, von Baeyer CL, Seshia S. Psychological interventions for headache in children and adolescents. Can J Neurol Sci. 2012;39(1):26-34.

35. Esposito M, Gallai B, Parisi L, et al. Self-concept evaluation and migraine without aura in childhood. Neuropsychiatr Dis Treat. 2013;9: 1061-1066.

36. Esposito M, Ruberto M, Gimigliano F, et al. Effectiveness and safety of Nintendo Wii Fit Plus ${ }^{\mathrm{TM}}$ training in children with migraine without aura: a preliminary study. Neuropsychiatr Dis Treat. 2013;9:1803-1810.

37. Esposito M, Roccella M, Gallai B, et al. Maternal personality profile of children affected by migraine. Neuropsychiatr Dis Treat. 2013;9: 1351-1358.

38. Esposito M, Marotta R, Gallai B, et al. Temperamental characteristics in childhood migraine without aura: a multicenter study. Neuropsychiatr Dis Treat. 2013;9:1187-1192.

39. Arruda MA, Guidetti V, Galli F, Albuquerque RC, Bigal ME. Migraine, tension-type headache, and attention-deficit/hyperactivity disorder in childhood: a population-based study. Postgrad Med. 2010;122(5): 18-26.

40. Guidetti V, Galli F, Sheftell F. Headache attributed to psychiatric disorders. Handb Clin Neurol. 2010;97:657-662.

41. Galli F, Canzano L, Scalisi TG, Guidetti V. Psychiatric disorders and headache familial recurrence: a study on 200 children and their parents. J Headache Pain. 2009;10(3):187-197.

42. Bruni O, Russo PM, Ferri R, Novelli L, Galli F, Guidetti V. Relationships between headache and sleep in a non-clinical population of children and adolescents. Sleep Med. 2008;9(5):542-548.

43. Galli F, D'Antuono G, Tarantino S, et al. Headache and recurrent abdominal pain: a controlled study by the means of the Child Behaviour Checklist (CBCL). Cephalalgia. 2007;27(3):211-219.

44. Guidetti V, Galli F. Psychiatric comorbidity in chronic daily headache: pathophysiology, etiology, and diagnosis. Curr Pain Headache Rep. 2002;6(6):492-497.

45. Guidetti V, Galli F, Cerutti R, Fortugno S. "From 0 to 18 ": what happens to the child and his headache? Funct Neurol. 2000;15 Suppl 3: $122-129$.

46. Guidetti V, Galli F, Fabrizi P, et al. Headache and psychiatric comorbidity: clinical aspects and outcome in an 8-year follow-up study. Cephalalgia. $1998 ; 18(7): 455-462$. 
47. Lambert GA, Zagami AS. The mode of action of migraine triggers: a hypothesis. Headache. 2009;49(2):253-275.

48. Messlinger K. Migraine: where and how does the pain originate? Exp Brain Res. 2009;196(1):179-193.

49. Parsons AA, Strijbos PJ. The neuronal versus vascular hypothesis of migraine and cortical spreading depression. Curr Opin Pharmacol. 2003;3(1):73-77.

50. Hamel E. Serotonin and migraine: biology and clinical implications. Cephalalgia. 2007;27(11):1293-1300.

51. Akerman S, Goadsby PJ. Dopamine and migraine: biology and clinical implications. Cephalalgia. 2007;27(11):1308-1314.

52. Anttila V, Stefansson H, Kallela M, et al; International Headache Genetics Consortium. Genome-wide association study of migraine implicates a common susceptibility variant on 8q22.1. Nat Genet. 2010;42(10):869-873.

53. Purves D, Augustine GJ, Fitzpatrick D, et al, editors. Neuroscience. 2nd ed. Sunderland, MA: Sinauer Associates; 2001.

54. Kai-Kai MA, Howe R. Glutamate-immunoreactivity in the trigeminal and dorsal root ganglia, and intraspinal neurons and fibres in the doral horn of the rat. Histochem J. 1991;23(4):171-179.

55. McKenna MC. The glutamate-glutamine cycle is not stoichiometric: fates of glutamate in brain. J Neurosci Res. 2007;85(15):3347-3358.

56. Sano Y, Inamura K, Miyake A, et al. A novel two-pore domain $\mathrm{K}+$ channel, TRESK, is localized in the spinal cord. $J$ Biol Chem. 2003;278(30):27406-27412.

57. Lafrenière RG, Rouleau GA. Migraine: role of the TRESK two-pore potassium channel. Int J Biochem Cell Biol. 2011;43(11):1533-1536.

58. Wood H. Migraine: familial migraine with aura is associated with a mutation in the TRESK potassium channel. Nat Rev Neurol. 2010;6(12):643

59. D'Andrea G, Leon A. Pathogenesis of migraine: from neurotransmitters to neuromodulators and beyond. Neurol Sci. 2010;31 Suppl 1:S1-S7.

60. Cannon SC. Physiologic principles underlying ion channelopathies. Neurotherapeutics. 2007;4(2):174-183.

61. Sano Y, Inamura K, Miyake A, et al. A novel two-pore domain $\mathrm{K}+$ channel, TRESK, is localized in the spinal cord. $J$ Biol Chem. 2003;278(30):27406-27412.

62. Liu C, Au JD, Zou HL, Cotten JF, Yost CS. Potent activation of the human tandem pore domain $\mathrm{K}$ channel TRESK with clinical concentrations of volatile anesthetics. Anesth Analg. 2004;99(6):1715-1722.

63. Lafrenière RG, Cader MZ, Poulin JF, et al. A dominant-negative mutation in the TRESK potassium channel is linked to familial migraine with aura. Nat Med. 2010;16(10):1157-1160.

64. Berman HM, Westbrook J, Feng Z, et al. The Protein Data Bank. Nucleic Acids Res. 2000;28(1):235-242.

65. Eswar N, Eramian D, Webb B, Shen MY, Sali A. Protein structure modeling with MODELLER. Methods Mol Biol. 2008;426: 145-159.

66. Colovos C, Yeates TO. Verification of protein structures: patterns of nonbonded atomic interactions. Protein Sci. 1993;2(9):1511-1519.

67. Melo F, Devos D, Depiereux E, Feytmans E. ANOLEA: a www server to assess protein structures. Proc Int Conf Intell Syst Mol Biol. 1997;5: $187-190$.

68. Laskowski RA, MacArthur MW, Moss DS, Thornton JM. PROCHECK: a program to check the stereochemical quality of protein structures. J Appl Cryst. 1993;26:283-291.
69. Lovell SC, Davis IW, Arendall WB 3rd, et al. Structure validation by Calpha geometry : phi,psi and Cbeta deviation. Proteins. 2002;50(3): 437-450.

70. Chen VB, Arendall WB 3rd, Headd JJ, et al. MolProbity: all-atom structure validation for macromolecular crystallography. Acta Crystallogr D Biol Crystallogr. 2010;66(Pt 1):12-21.

71. Emsley P, Lohkamp B, Scott WG, Cowtan K. Features and development of Coot. Acta Crystallogr D Biol Crystallogr. 2010;66(Pt 4): 486-501.

72. Pettersen EF, Goddard TD, Huang CC, et al. UCSF Chimera - a visualization system for exploratory research and analysis. J Comput Chem. 2004;25(13):1605-1612.

73. Pedretti A, Villa L, Vistoli G. VEGA - an open platform to develop chemo-bio-informatics applications, using plug-in architecture and script programming. J Comput Aided Mol Des. 2004;18(3): $167-173$.

74. Mendelsohn LD. ChemDraw 8 Ultra, Windows and Macintosh versions. J Chem Inf Comput Sci. 2004;44(6):2225-2226.

75. Trott O, Olson AJ. AutoDock Vina: improving the speed and accuracy of docking with a new scoring function, efficient optimization, and multithreading. J Comput Chem. 2010;31(2):455-461.

76. Mishra NK, Raghava GP. Prediction of specificity and cross-reactivity of kinase inhibitors. Lett Drug Des Disc. 2011;8:223-228.

77. Bolton E, Wang Y, Thiessen PA, Bryant SH. PubChem: Integrated Platform of Small Molecules and Biological Activities. In: Annual Reports in Computational Chemistry, Volume 4. Washington, DC: American Chemical Society; 2008.

78. Wolber G, Langer T. LigandScout: 3-D pharmacophores derived from protein-bound ligands and their use as virtual screening filters. J Chem Inf Model. 2005;45(1):160-169.

79. Wallace AC, Laskowski RA, Thornton JM. LIGPLOT: a program to generate schematic diagrams of protein-ligand interactions. Protein Eng. 1996;8(2):127-134.

80. Machado-Alba JE, Morales-Plaza CD. Use of ergotamine: do physicians in Colombia know how to prescribe it? Neurologia. 2013;pii: S0213-4853(13)00153-9.

81. Zapalska-Pozarowska K, Szponar J, Górska A, Niewiedzioł M. [Ergotamine poisoning: a case study]. Przegl Lek. 2012;69(8):627-628. Polish.

82. Helsen V, Decoutere L, Spriet I, Fagard K, Boonen S, Tournoy J. Ergotamine-induced pleural and pericardial effusion successfully treated with colchicine. Acta Clin Belg. 2013;68(2):113-115.

83. Ramón-Carbajo C, Alvarez-Escudero R, Pascual J. [Rizatriptan: experience after 15 years of clinical use]. Rev Neurol. 2013;16(12): 549-555. Spanish.

84. Derry S, Rabbie R, Moore RA. Diclofenac with or without an antiemetic for acute migraine headaches in adults. Cochrane Database Syst Rev. 2013;4:CD008783.

85. Kirthi V, Derry S, Moore RA. Aspirin with or without an antiemetic for acute migraine headaches in adults. Cochrane Database Syst Rev. 2013;4:CD008041.

\section{Publish your work in this journal}

Drug Design, Development and Therapy is an international, peerreviewed open-access journal that spans the spectrum of drug design and development through to clinical applications. Clinical outcomes, patient safety, and programs for the development and effective, safe, and sustained use of medicines are a feature of the journal, which

\section{Dovepress}

has also been accepted for indexing on PubMed Central. The manuscript management system is completely online and includes a very quick and fair peer-review system, which is all easy to use. Visit http://www.dovepress.com/testimonials.php to read real quotes from published authors. 